\title{
EXISTENCE OF SOLUTIONS FOR A FAMILY OF POLYHARMONIC AND BIHARMONIC EQUATIONS
}

\author{
M. HESAARAKI AND B. RAESSI
}

Received 17 April 2005 and in revised form 28 September 2005

We consider a family of polyharmonic problems of the form $(-\Delta)^{m} u=g(x, u)$ in $\Omega$, $D^{\alpha} u=0$ on $\partial \Omega$, where $\Omega \subset \mathbb{R}^{n}$ is a bounded domain, $g(x, \cdot) \in L^{\infty}(\Omega)$, and $|\alpha|<m$. By using the fibering method, we obtain some results about the existence of solution and its multiplicity under certain assumptions on $g$. We also consider a family of biharmonic problems of the form $\Delta^{2} u+\left(\Delta \varphi+|\nabla \varphi|^{2}\right) \Delta u+2 \nabla \varphi \cdot \nabla \Delta u=g(x, u)$, where $\varphi \in C^{2}(\bar{\Omega})$, and $\Omega, g$, and the boundary condition are the same as above. For this problem, we prove the existence and multiplicity of solutions too.

\section{Introduction}

In this paper, we consider the following polyharmonic problem:

$$
\begin{aligned}
(-\Delta)^{m} u & =g(x, u), \quad \text { in } \Omega, \\
D^{\alpha} u & =0, \quad \text { on } \partial \Omega,
\end{aligned}
$$

where $\Omega \subset \mathbb{R}^{n}$ is a bounded domain, $g(x, u)=\sum \varepsilon_{j} g_{j}(x)|u|^{p_{j}-1} u+h(x)$, with $0 \leq g_{j} \in$ $L^{\infty}(\Omega), \varepsilon_{j} \in\{0,1,-1\}, 1<p_{1}<p_{2}<\cdots<p_{l}<(n+2 m) /(n-2 m),|\alpha|<m$, and $h \in$ $\left(H_{0}^{m}(\Omega)\right)^{*}$.

This problem has been studied before by many authors for special cases as follows.

(1) It is well known that for $m=1, g(x, u)=|u|^{p-1} u$, the problem (1.1)-(1.2) has a solution if $1<p<(n+2) /(n-2)$ and does not have any solution if $(n+2) /(n-2)<p$. The usual approaches to prove the existence or nonexistence of solutions in this case are mountain-pass lemma and Pohozaev identity, respectively; see [4, Sections 8.5.2 and 9.4.2]. Moreover, for the critical Sobolev exponents case, that is, $p=(n+2) /(n-2)$, Brézis and Nirenberg, by considering $g=f+u^{(n+2) /(n-2)}$, have proved that, if $f \equiv 0$, the problem has no solution in a starlike domain. They have also proved the existence of solutions for some cases of $f \not 0$, [3].

(2) For $\Omega=\mathbb{R}^{n}, g(x, u)=|u|^{p-1} u$, and $p=(n+2 m) /(n-2 m)$, Bartsch, Schneider, and Weth have established that (1.1) has a sequence of nodal finite-energy solutions, which is unbounded in $D^{m, 2}\left(\mathbb{R}^{n}\right)$, see [2]. 
3406 Solutions for polyharmonic and biharmonic equations

(3) Let $B$ be the unit ball in $\mathbb{R}^{n}$ and $\Omega=B$, moreover, suppose that $g(x, u)$ satisfies the following hypotheses.

(H1) The function $g$ is nonnegative, Borel measurable function on $B \times(0, \infty)$, which is continuous and nonincreasing with respect to the second variable.

(H2) For each $c>0$, the function $x \mapsto g\left(x, c(1-|x|)^{m}\right) /(1-|x|)^{m-1}$ is in $K_{m, n}$, where $K_{m, n}$ is a set of Borel measurable functions, such as $\varphi$, which are defined on $B$, and satisfy the condition

$$
\lim _{\alpha \rightarrow 0}\left(\sup _{x \in B} \int_{B \cap B(x, \alpha)}\left(\frac{1-|y|}{1-|x|}\right)^{m} G_{m, n}(x, y)|\varphi(y)| d y\right)=0 .
$$

Here $G_{m, n}$ is the green function of $(-\Delta)^{m}$ on the unit ball $B$.

(H3) For each $c>0, g(\cdot, c)$ is positive on a set of positive measures.

Under the above assumption, Mâagli et al. in [10] have proved the following theorem.

Theorem 1.1. Assume that (H1)-(H3) hold, then the problem (1.1)-(1.2) has a positive continuous solution. Moreover, there exist two positive constants $a$ and $b$ such that for each $x \in B$,

$$
a(1-|x|)^{m} \leq u(x) \leq b(1-|x|)^{m-1} .
$$

(4) Grunau in [5] studied the growth conditions that imply the existence of a strong solution for the following type of problems:

$$
\begin{gathered}
L u+g(x, u)=f(x), \quad \text { in } \Omega, \\
D^{\alpha} u=0, \quad \text { on } \partial \Omega,
\end{gathered}
$$

where $|\alpha|<m, L=(-\Delta)^{m}$, and $u \mapsto g(\cdot, u)$ exceeds the controllable growth rate $u^{(n+2 m) /(n-2 m)}$. Recently in [6] this result has been extended to

$$
L=\left(-\sum_{i, j=1}^{n} a_{i j} \frac{\partial^{2}}{\partial x_{i} \partial x_{j}}\right)^{m}+\sum_{|\alpha| \leq 2 m-1} b_{\alpha}(x) D^{\alpha},
$$

with $a_{i j} \in \mathbb{R}, \sum_{i, j=1}^{n} a_{i j} \xi_{i} \xi_{j} \geq c|\xi|^{2}, b_{\alpha} \in C^{|\alpha|, \gamma}(\bar{\Omega})$, and $L$ is assumed to be coercive which means that for some $c>0$ and all $u \in W_{0}^{m, 2}(\Omega) \cap C^{2 m}(\bar{\Omega})$, one has

$$
\int_{\Omega} u L u d x \geq c\|u\|_{W^{m, 2}(\Omega)}^{2}
$$

By assuming that $g$ and $\Omega$ are sufficiently smooth, Grunau and Sweers in [6] proved the following theorem.

Theorem 1.2. Fix $n \geq 2 m$ and suppose that $g$ satisfies the sign condition, $u g(\cdot, u) \geq 0$, for all $u \in \mathbb{R}$, and the one-sided growth condition

$$
g(x, u) \geq-c\left(1+|u|^{\sigma}\right)
$$


with

$$
\begin{aligned}
& \sigma=1 \quad \text { if } 6 m<n, \\
& \sigma<\frac{4 m}{n-2 m} \quad \text { if } 2 m<n<6 m, \\
& \sigma<\infty \quad \text { if } n=2 m,
\end{aligned}
$$

then for every $f \in C^{\alpha}(\bar{\Omega})$, the problem (1.5) has a solution $u \in C^{2 m, \alpha}(\Omega) \cap W_{0}^{m, 2}(\Omega)$.

Remark 1.3. For the following two-sided growth condition (instead of (1.8)),

$$
\begin{aligned}
& g(x, u) \geq-c\left(1+|u|^{\tau}\right) \quad \text { for } u \leq 0, \\
& g(x, u) \leq c\left(1+|u|^{\tau}\right) \quad \text { for } u \geq 0,
\end{aligned}
$$

with $\tau \leq(n+2 m) /(n-2 m)$, the existence of a weak solution in $W_{0}^{m, 2}(\Omega)$ follows from the coercivity of $L$. Moreover, for $\tau<(n+2 m) /(n-2 m)$, a linear argument, bootstrapping between Sobolev imbedding and regularity theory, see [1], shows existence of a strong solution $u \in C^{2 m}(\bar{\Omega})$ as well as regularity of any weak solution. Luckhaus [9] also proved for general elliptic operators that all solutions of (1.5) are classic, that is, $u \in C^{2 m}(\bar{\Omega})$, whenever (1.10) holds with $\tau \leq(n+2 m) /(n-2 m)$.

Remark 1.4. For $m=1$, no controllable growth conditions are needed. Here the maximum principle together with the sign condition for $g$ gives an $L^{\infty}$ bound to start the bootstrapping. For $m=2$, Tomi in [20] obtained a classical solution by using the maximum principle for an auxiliary function like $a(\Delta u)^{2}+G(u)$, where $G^{\prime}=g$ and $a \in \mathbb{R}$.

Remark 1.5. These approaches do not work for general higher-order elliptic equations with zero Dirichlet boundary conditions. Not only no maximum principle on general domains exists, but also the restriction to a level set defines a new nonzero Dirichlet problem. By exploiting the Green function estimates on balls, a local maximum principle can however be proved.

This paper is organized as follows. In Section 2, we consider the equation

$$
(-\Delta)^{m} u=f(x)|u|^{p-1} u,
$$

with the boundary condition (1.2) and $f \in L^{\infty}(\Omega)$. We prove the existence of infinitely many solutions. In Section 3, we consider the equation

$$
(-\Delta)^{m} u=\varepsilon f(x)|u|^{p-1} u+\delta g(x)|u|^{q-1} u+h(x),
$$

with the boundary condition (1.2) and $0 \leq f, g \in L^{\infty}(\Omega)$. Then we prove that for small values of $\|h\|_{*}$, this problem has at least one solution or three solutions depend on $\varepsilon, \delta \in$ $\{1,-1\}$. In Section 4 , we consider the biharmonic equation

$$
\Delta^{2} u+\left(\Delta \varphi+|\nabla \varphi|^{2}\right) \Delta u+2 \nabla \varphi \cdot \nabla \Delta u=\varepsilon f(x)|u|^{p-1} u+\delta g(x)|u|^{q-1} u+h(x),
$$

with the boundary condition (1.2), for $|\alpha|<4, \varphi \in C^{2}(\bar{\Omega})$, and $f, g, h, \varepsilon, \delta$ as above. We prove the existence of one solution or multiple solutions. 
3408 Solutions for polyharmonic and biharmonic equations

\section{A special case}

Let $\Omega$ be a bounded domain in $\mathbb{R}^{n}$ and $n, m \in \mathbb{N}$. In this section and the next one, we let

$$
p^{*}:= \begin{cases}\frac{n+2 m}{n-2 m}, & \text { for } n>2 m \\ \infty, & \text { for } n \leq 2 m\end{cases}
$$

Let $H:=H^{m}(\Omega)$ and $H_{0}:=H_{0}^{m}(\Omega)$ be the Sobolev spaces, where $H_{0}$ is equipped with the scalar product

$$
(u, v):= \begin{cases}\int_{\Omega} \Delta^{m / 2} u \cdot \Delta^{m / 2} v, & m \text { even } \\ \int_{\Omega} \nabla \Delta^{(m-1) / 2} u \cdot \nabla \Delta^{(m-1) / 2} v, & m \text { odd }\end{cases}
$$

Consider the problem

$$
\begin{gathered}
(-\Delta)^{m} u=f(x)|u|^{p-1} u, \quad \text { in } \Omega, \\
D^{\alpha} u=0, \quad \text { on } \partial \Omega,
\end{gathered}
$$

where $1<p<p^{*}, f \in L^{\infty}(\Omega), f=f_{1}-f_{2}, f_{i} \geq 0$, and $f_{1}$ is not identically zero. The Euler functional of (2.3) is given by

$$
I[u]:=\frac{1}{2}(u, u)-\frac{1}{p+1} \int_{\Omega} f(x)|u|^{p+1} d x
$$

where $(\cdot, \cdot)$ is the scalar product on $H_{0}$, which is defined by $(2.2)$. It is known that a conditional critical point of $I$ corresponds to a weak solution of the boundary-value problem (2.3). Let us split the function $u \in H_{0}$ as follows:

$$
u=r v(x), \quad r \in \mathbb{R} \backslash\{0\}, v \in \Gamma,
$$

where $\Gamma=\left\{v \in H_{0}: \int_{\Omega} f(x)|v|^{p+1} d x>0,(v, v)=1\right\}$. Now if we substitute from (2.5) into (2.4), then we obtain

$$
E(r, v):=I[r v]=\frac{r^{2}}{2}-\frac{|r|^{p+1}}{p+1} \int_{\Omega} f|v|^{p+1} d x
$$

Following the fibering method suggested in $[12,13,14]$, we obtain

$$
I^{\prime}[u]=0 \Longleftrightarrow\left\{\begin{array}{l}
E_{r}^{\prime}(r, v)=0, \\
E_{v}^{\prime}(r, v)=0,
\end{array}\right.
$$

where $E_{v}^{\prime}(r, v)$ is the derivative of $E(r, v)$ in the tangential direction on the boundary of the unit sphere in $H_{0}$. The equation $E_{r}^{\prime}(r, v)=0$ plays the same role as the bifurcation equation in the classical approach. Therefore, it is referred to as the bifurcation equation 
in the fibering method as well. In this case, the bifurcation equation acquires the form $r-r|r|^{p-1} \int_{\Omega} f|v|^{p+1} d x=0$. This equation has three roots:

$$
r_{1}(v)=0, \quad r_{2}(v)=-r_{3}(v)=\left(\int_{\Omega} f|v|^{p+1} d x\right)^{-2 /(p-1)} .
$$

The nontrivial roots give rise to the functional

$$
E(v):=E\left(r_{i}(v), v\right)=\left(\frac{1}{2}-\frac{1}{p+1}\right)\left(\int_{\Omega} f|v|^{p+1} d x\right)^{-2 /(p-1)}, \quad i=2,3 .
$$

If we set $\widetilde{E}(v)=\int_{\Omega} f|v|^{p+1} d x$, then we see that $\widetilde{E}(v)$ and $E(v)$ have the same conditional critical points. Moreover, $\widetilde{E}(v)$ is strictly positive and is bounded from above for all $v \in \Gamma$. In order to see this notice that for $0 \leq p<p^{*}$, the embeddings $H_{0}(\Omega) \subset L^{p+1}(\Omega)$ are compact continuous and there exists a $c>0$ such that

$$
\|u\|_{p+1} \leq c\|u\|_{H_{0}}
$$

thus

$$
0<\tilde{E}(v)=\int_{\Omega} f|v|^{p+1} d x \leq\|f\|_{\infty} c^{p+1}<\infty .
$$

Now we arrive to the following theorem.

Theorem 2.1. The problem (2.3) has at least one weak solution. In other words, there exists a point $v_{0} \in H_{0}$, which is a maximum point for the problem

$$
M=\sup \{\tilde{E}(v) \mid v \in \Gamma\} .
$$

Proof. Let $\left\{v_{i}\right\} \subset \Gamma$ be a maximizing sequence for $M$, that is, $v_{i} \in \Gamma$ and $\widetilde{E}\left(v_{i}\right) \rightarrow M$. From compactness of the embedding $H_{0} \subset L^{p+1}(\Omega)$, for $1<p<p^{*}$, and boundedness of $\left\{v_{i}\right\}$ in $H_{0}$, that is, $\left(v_{i}, v_{i}\right)=1$, and by weak continuity of the functional $u \mapsto \int_{\Omega} f(x)|u|^{p+1} d x$, it follows that there exists a subsequence $\left\{v_{i_{j}}\right\}$ of $\left\{v_{i}\right\}$ and $v_{0} \in H_{0}$ such that $v_{i_{j}}$ is weakly convergent to $v_{0}$ in $H_{0}$, and strongly convergent to the same $v_{0}$ in $L^{p+1}(\Omega)$. Thus

$$
\widetilde{E}\left(v_{i_{j}}\right) \longrightarrow \widetilde{E}\left(v_{0}\right)=M>0 .
$$

We claim that $v_{0} \neq 0$. Indeed, if $v_{0}=0$, then $\widetilde{E}\left(v_{0}\right)=0$, which contradicts with $M>0$. Now it remains to show that $v_{0} \in \Gamma$. By lower semicontinuity of the norm, we have

$$
\left(v_{0}, v_{0}\right) \leq \liminf \left(v_{i_{j}}, v_{i_{j}}\right)=1 \text {. }
$$

If we suppose $0<\left(v_{0}, v_{0}\right)<1$, then for a suitable $k>1,\left(k v_{0}, k v_{0}\right)=1$ and $\widetilde{E}\left(k v_{0}\right)=k^{p+1} M>$ $M$, which contradicts with the definition of $M$. The proof of the theorem is completed.

Remark 2.2. For $v_{0}$ as above, we have $\left|v_{0}\right| \in \Gamma$ and also $\widetilde{E}\left(\left|v_{0}\right|\right)=\widetilde{E}\left(v_{0}\right)=M_{0}$. Therefore, we have $u_{0}(x)=\left(\int_{\Omega} f\left|v_{0}\right|^{p+1} d x\right)^{-2 /(p-1)}\left|v_{0}(x)\right|$, as a positive solution of (2.3). 
3410 Solutions for polyharmonic and biharmonic equations

Remark 2.3. Notice that Lyusternik-Shnirelman theory can be applied to the functional $\widetilde{E}(v)$ on the unit sphere in $H_{0}$. According to this theory, $\widetilde{E}(v)$ has countably many alternating conditional critical points $v_{1}, v_{2}, \ldots, v_{k}, \ldots$ such that $\|v\|_{H_{0}}=1$ and $\tilde{E}\left(v_{k}\right) \rightarrow 0$ as $k \rightarrow \infty$. Therefore, the problem (2.3) has countably many alternating solutions $\left\{u_{k}\right\}$ : $u_{k}(x)= \pm\left(\int_{\Omega} f\left|v_{k}\right|^{p+1} d x\right)^{-2 /(p-1)} v_{k}(x)$.

\section{The general case}

Consider the problem

$$
\begin{gathered}
(-\Delta)^{m} u=\varepsilon f(x)|u|^{p-1} u+h(x), \quad \text { in } \Omega, \\
D^{\alpha} u=0, \quad \text { on } \partial \Omega,
\end{gathered}
$$

where $1<p<p^{*}, \varepsilon \in\{1,-1\}, h \in H_{0}^{*}$ (dual space of $H_{0}$ ), and $f \in L^{\infty}(\Omega)$ with $f \geq 0$.

The above problem for the case $m=1$ has been studied before for existence of solutions by many authors. Tarantello in [19] considered the problem for $\varepsilon f(x)=1$, then he showed that for the limiting exponent $p=(n+2) /(n-2)$, the problem has two solutions in $H_{0}^{1}(\Omega)$, if $f \in H^{-1}(\Omega)$ and $f \not 0$.

In [8], Li and Liu considered the above problem for $m=1$ and $|\varepsilon|$ small, then they proved the existence of multiple solutions for the problem under some reasonable assumptions.

In [18], also Rădulescu and Smets considered the problem with critical exponent and a small inhomogeneous term. Under some conditions, two solutions are found.

In the following theorem and Theorem 3.4, we consider the above problem for the existence of solution, if $m \geq 1$.

TheOREm 3.1. The problem (3.1) has at least three solutions for $\varepsilon=1$ and sufficiently small $\|h\|_{*}$. This problem has one solution for $\varepsilon=-1$ and every $h$ in $H_{0}^{*}$.

Proof. The Euler functional of this problem is given by

$$
I[u]:=\frac{1}{2}(u, u)-\frac{\varepsilon}{p+1} \int_{\Omega} f(x)|u|^{p+1} d x-\int_{\Omega} h u d x .
$$

Let $u=r v(x), r \in \mathbb{R} \backslash\{0\}, v \in \Gamma$, where $\Gamma=\left\{v \in H_{0} \mid(v, v)=1\right\}$ and as before we define $E(r, v):=I[r v]$. Then $u=r v$ is a critical point of $I$, if $E_{r}^{\prime}=E_{v}^{\prime}=0$. But

$$
E_{r}^{\prime}=r-\varepsilon r|r|^{p-1} \int_{\Omega} f|v|^{p+1} d x-\int_{\Omega} h v d x
$$

Now let $\psi(r)=r-\left.\varepsilon r|r|\right|^{p-1} \int_{\Omega} f|v|^{p+1} d x$. Here we consider two different cases as follows. Case $1(\varepsilon=1)$. In this case, the graph of $\psi$ looks like the graph of $y=x-x^{3}$. Thus the equation $E_{r}^{\prime}=0$ has three zeros $r_{i}(v), i=1,2,3$, if $h$ satisfies

$$
\left|\int_{\Omega} h v d x\right| \leq \operatorname{local} \max \psi(r)
$$

for all $v \in \Gamma$, that is, $\|h\|_{*} \leq(1 / c)$ local max $\psi(r)$, where $c$ is a constant defined in (2.10). Suppose that $r_{2}(v)>0$ and $r_{3}(v)<0$ are the local maximum points of $E(r, v)$, and $r_{1}(v)$ is 
its local minimum point. Notice that $r_{1}(v) \int_{\Omega} h v d x \geq 0$. Let

$$
E_{i}(v):=\frac{\left|r_{i}(v)\right|^{2}}{2}-\frac{\left|r_{i}(v)\right|^{p+1}}{p+1} \cdot \int_{\Omega} f|v|^{p+1} d x-r_{i}(v) \cdot \int_{\Omega} h v d x, \quad i=1,2,3 .
$$

We know that $r_{i}(v), i=1,2,3$, are weakly continuous functionals. From (3.3) we have $\left|r_{i}(v)\right| \leq c\left(\|f\|_{\infty},\|h\|_{*}\right)$ for $v \in \Gamma$, and from (3.5) we have $\left|E_{i}(v)\right| \leq c\left(\|f\|_{\infty},\|h\|_{*}\right)$. Moreover, $E_{1}(v) \leq 0$. In order to complete the proof in this case, we have the following lemma.

Lemma 3.2. There exists $v_{0}^{i} \in H_{0}$, which minimizes the value of $E_{i}(v)$ on $\Gamma$, for $i=1,2,3$.

Proof. Let

$$
m_{i}=\inf \left\{E_{i}(v) \mid v \in \Gamma\right\},
$$

and $\left\{v_{l}^{i}\right\} \subset \Gamma$, be a minimizing sequence for $E_{i}(v)$. As we see in the proof of Theorem 3.1, $\left\{v_{l}^{i}\right\}$ is bounded in $H_{0}$. Therefore, there exists a subsequence $\left\{v_{l_{j}}^{i}\right\}$ of $\left\{v_{l}^{i}\right\}$ and $v_{0}^{i} \in H_{0}$ such that $v_{l_{j}}^{i} \rightarrow v_{0}^{i}$ weakly in $H_{0}$ and $v_{l_{j}}^{i} \rightarrow v_{0}^{i}$ strongly in $L^{p+1}(\Omega)$, for $1<p<p^{*}$. Hence, by continuity of $r_{i}(v)$ we must have $r_{i}\left(v_{l_{j}}^{i}\right) \rightarrow r_{i}\left(v_{0}^{i}\right)$ and $E_{i}\left(v_{l_{j}}^{i}\right) \rightarrow E_{i}\left(v_{0}^{i}\right)=m_{i}$. Now we show that $v_{0}^{i} \neq 0$. Suppose $v_{0}^{i}=0$. Then $E_{i}\left(v_{0}^{i}\right)=r_{i}^{2}\left(v_{0}^{i}\right) / 2 \geq 0$, which is a contradiction to $m_{i}<$ 0 . It remains to show that $v_{0}^{i} \in \Gamma$, that is, $\left(v_{0}^{i}, v_{0}^{i}\right)=1$. Notice that by lower semicontinuity of the norm we have $\left(v_{0}^{i}, v_{0}^{i}\right) \leq \liminf \left(v_{l_{j}}^{i}, v_{l_{j}}^{i}\right)=1$. Suppose that we have $0<\left(v_{0}^{i}, v_{0}^{i}\right)<1$. Let $\lambda_{0}=\left(v_{0}^{i}, v_{0}^{i}\right)^{-1}>1$. For $0<\lambda<\lambda_{0}$, we consider the function $E_{i}\left(r_{i}\left(\lambda v_{0}^{i}\right), \lambda v_{0}^{i}\right)$. For this function, we can write

$$
\begin{aligned}
\frac{d E_{i}}{d \lambda}= & \left(r_{i}\left(\lambda v_{0}^{i}\right)-r_{i}\left(\lambda v_{0}^{i}\right)\left|r_{i}\left(\lambda v_{0}^{i}\right)\right|^{p-1} \int_{\Omega} f\left|\lambda v_{0}^{i}\right|^{p+1} d x-\int_{\Omega} h\left(\lambda v_{0}^{i}\right) d x\right) \cdot \frac{d r_{i}}{d \lambda} \\
& -\frac{1}{\lambda}\left(r_{i}\left(\lambda v_{0}^{i}\right)\left|r_{i}\left(\lambda v_{0}^{i}\right)\right|^{p-1} \int_{\Omega} f\left|\lambda v_{0}^{i}\right|^{p+1} d x+r_{i}\left(\lambda v_{0}^{i}\right) \int_{\Omega} h\left(\lambda v_{0}^{i}\right) d x\right) \\
= & -\frac{1}{\lambda}\left(r_{i}\left(\lambda v_{0}^{i}\right)\right)^{2}<0 .
\end{aligned}
$$

Therefore, $E_{i}$ is a decreasing function of $\lambda$. Hence, we must have $E_{i}\left(r_{i}\left(\lambda_{0} v_{0}^{i}\right), \lambda_{0} v_{0}^{i}\right)<E_{i}\left(r_{i}\left(v_{0}^{i}\right)\right.$, $v_{0}^{i}$ ), which is a contradiction to the definition of $m_{i}$. By considering the above lemma, the proof in Case 1 is complete.

Case $2(\varepsilon=-1)$. In this case, the graph of $\psi$ looks like $y=x+x^{3}$, it follows that the equation $E_{r}^{\prime}=0$ has exactly one root $r(v)$, for every $h$ in $H_{0}^{*}$. Let $E(v)=E(r(v), v)$. Notice that $E(v) \leq 0$ and $E$ is bounded from below. Now by using some arguments similar to the proof of Lemma 3.2, we can prove the following lemma, which completes the proof in Case 2.

LEMMA 3.3. If

$$
m:=\inf \{E(v) \mid v \in \Gamma\}
$$

then there is $v_{0} \in \Gamma$, which minimizes $m$. The proof of Theorem 3.1 is completed. 
3412 Solutions for polyharmonic and biharmonic equations

Now consider the problem

$$
\begin{gathered}
(-\Delta)^{m} u=\varepsilon f(x)|u|^{p-1} u+\delta g(x)|u|^{q-1} u+h(x), \quad \text { in } \Omega, \\
D^{\alpha} u=0, \quad \text { on } \partial \Omega,
\end{gathered}
$$

where $1<p<q<p^{*}, \varepsilon, \delta \in\{1,-1\}, f, g \geq 0$, and $h \in H_{0}^{*}(\Omega)$. We have the following theorem about this problem.

Theorem 3.4. The problem (3.9) has

(1) one solution for every $h$ and $\varepsilon=\delta=-1$,

(2) three solutions for sufficiently small $\|h\|_{*}$ and $\varepsilon=\delta=1$,

(3) three solutions for sufficiently small $\|h\|_{*}$ and $\varepsilon=-\delta=1$.

Proof. The Euler functional for this problem is given by

$$
I[u]:=\frac{1}{2}(u, u)-\frac{\varepsilon}{p+1} \int_{\Omega} f(x)|u|^{p+1} d x-\frac{\delta}{q+1} \int_{\Omega} g(x)|u|^{q+1} d x-\int_{\Omega} h u d x .
$$

As before we let $u=r v(x), r \in \mathbb{R} \backslash\{0\}, v \in \Gamma$, where $\Gamma=\left\{u \in H_{0} \mid(u, u)=1\right\}$. Let

$$
\begin{aligned}
E(r, v): & =I[r v]=\frac{r^{2}}{2}-\frac{\varepsilon|r|^{p+1}}{p+1} \int_{\Omega} f|v|^{p+1} d x-\frac{\delta|r|^{q+1}}{q+1} \int_{\Omega} g|v|^{q+1} d x-r \int_{\Omega} h v d x, \\
\psi(r, v) & =\frac{d}{d r} E(r, v)+\int_{\Omega} h v d x \\
& =r-\varepsilon r|r|^{p-1} \int_{\Omega} f|v|^{p+1} d x-\delta r|r|^{q-1} \int_{\Omega} g|v|^{q+1} d x .
\end{aligned}
$$

Again we consider three cases as follows.

Case $1(\varepsilon=\delta=-1)$. In this case,

$$
\psi(r, v)=r+r|r|^{p-1} \int_{\Omega} f|v|^{p+1} d x+r|r|^{q-1} \int_{\Omega} g|v|^{q+1} d x
$$

and $d \psi / d r \geq 0$, then for every $h \in H_{0}^{*}$ and $v \in \Gamma$, the equation $E_{r}^{\prime}(r, v)=0$ has exactly one root $r(v)$. Moreover, for fixed $v$, the graph of $E(r, v)$ looks like the graph of $y=x(x-$ $2 r(v))$. Then $E(v)=E(r(v), v) \leq 0$. Now as before $r(v)$ and $E(v)$ are continuous functions of $v$, and $E(v)$ is bounded from below for $v \in \Gamma$. Thus $E$ has a minimizer $v_{0} \in H_{0}$. As before, by considering the lower semicontinuity of the norm, the zero Dirichlet boundary condition, and an argument similar to the proof of Lemma 3.2, we must have $v_{0} \in \Gamma$ and $u=r\left(v_{0}\right) v_{0}$ is a solution of the problem (3.9).

Case $2(\varepsilon=\delta=1)$. In this case,

$$
\psi(r, v)=r-r|r|^{p-1} \int_{\Omega} f|v|^{p+1} d x-r|r|^{q-1} \int_{\Omega} g|v|^{q+1} d x
$$

and the graphs of $\psi(r, v)$ and $E(r, v)$, for fixed $v \in \Gamma$, look like the graphs of $y=x-x^{3}$ and $y=x^{2}-x^{4}-r(v) x$, respectively. Now, for $\|h\|_{*}<(1 / c)$ local $\max \psi(r)$, the equation 
$\psi(r, v)=\int_{\Omega} h v d x$ has exactly three solutions $r_{i}(v), i=1,2,3$, where $c$ is the constant which is defined by (2.10). Let $E_{i}(v)=E\left(r_{i}(v), v\right)$, then $r_{i}(v)$ and $E_{i}(v)$ are weakly continuous functions. Notice that $E_{i}(v)$ is a bounded functional for $v \in \Gamma$. Thus the problem $m_{i}=$ $\inf \left\{E_{i}(v) \mid v \in \Gamma\right\}$ has a minimizer $v_{0}^{i} \in \Gamma$. Therefore, we have three solutions $u_{i}(x)=$ $r_{i}\left(v_{0}^{i}\right) v_{0}^{i}(x)$ for the problem (3.9).

Case $3(\varepsilon=-\delta=1)$. In this case,

$$
\psi(r, v)=r+r|r|^{p-1} \int_{\Omega} f|v|^{p+1} d x-r|r|^{q-1} \int_{\Omega} g|v|^{q+1} d x
$$

and the graphs of $\psi(r, v)$ and $E(r, v)$, for fixed $v \in \Gamma$, look like the graphs of $y=x+x^{2}-x^{3}$ and $y=x^{2}+|x|^{3}-x^{4}-a x$, respectively. Therefore, the equation $E_{r}^{\prime}=0$, for sufficiently small values of $\|h\|_{*}$, has exactly three roots and the problem (3.9) has three solutions in this case too.

Remark 3.5. Let $p=1, q=(n+2 m) /(n-2 m), f \equiv \lambda, g \equiv 1, \varepsilon=\delta=1, h \equiv 0$, then for $\Omega=B$, the unit ball in $\mathbb{R}^{n},(3.9)$ reduced to the problem

$$
\begin{gathered}
(-\Delta)^{m} u=\lambda u+|u|^{q-1} u, \quad \text { in } B, \\
D^{\alpha} u=0, \quad \text { on } \partial B .
\end{gathered}
$$

Due to the criticality of exponent $q$, a nontrivial solution to (3.15) may exist at most for $\lambda>0$, if $m=1$ [11], and for $\lambda \geq 0$, if $m>1$ [16]. We define after Pucci and Serrin [17] that the dimension $n$ is called critical (with respect to the boundary-value problem (3.15)) if and only if there is a positive bound $\Lambda>0$ such that a necessary condition for existence of a nontrivial radial solution to (3.15) is $\lambda>\Lambda$. Pucci and Serrin [17] showed that for any $m$, the dimension $n=2 m+1$ is critical and moreover that $n=5,6,7$ are critical in the fourth-order problem, $m=2$. They conjectured the following.

Conjecture of Pucci and Serrin. The critical dimensions for the boundary-value problem (3.15) are precisely $n=2 m+1, \ldots, 4 m+1$.

Remark 3.6. Consider the following polyharmonic problem:

$$
\begin{aligned}
(-\Delta)^{m} u & =g(x, u), \quad \text { in } \Omega, \\
D^{\alpha} u & =0, \quad \text { on } \partial \Omega,
\end{aligned}
$$

where $\Omega \subset \mathbb{R}^{n}$ is a bounded domain, $g(x, u)=\sum \varepsilon_{j} g_{j}(x)|u|^{p_{j-1}} u+h(x)$, with $0 \leq g_{j} \in$ $L^{\infty}(\Omega), \varepsilon_{j} \in\{0,1,-1\}, 1<p_{1}<p_{2}<\cdots<p_{l}<p^{*},|\alpha|<m$, and $h \in\left(H_{0}^{m}(\Omega)\right)^{*}$. Let

$$
I[u]:=\frac{1}{2}(u, u)-\sum_{j=1}^{l} \frac{\varepsilon_{j}}{p_{j}+1} \int_{\Omega} g_{j}|u|^{p_{j}+1} d x-\int_{\Omega} h u d x,
$$

and let $E(r, v):=I[r v]$ and $\psi(r, v)=(d / d r) E(r, v)+\int_{\Omega} h v d x$, where $v \in \Gamma$. Similar to the above cases, we can show that for every $v \in \Gamma$ and $h \in H_{0}^{*}$, with sufficiently small norm, the equation $E_{r}^{\prime}=0$ has at least one nonzero root. Therefore, the problem (3.16) has at least one solution. 
3414 Solutions for polyharmonic and biharmonic equations

\section{Biharmonic problem}

Let $\Omega$ be a bounded smooth domain in $\mathbb{R}^{n}$. In this section, we let $q^{*}=(n+4) /(n-4)$ if $n>4$ and $q^{*}=\infty$ otherwise. Let us now turn to the biharmonic problem

$$
\begin{gathered}
\Delta^{2} u+\left(\Delta \varphi+|\nabla \varphi|^{2}\right) \Delta u+2 \nabla \varphi \cdot \nabla \Delta u=f(x)|u|^{p-1} u, \quad \text { in } \Omega, \\
D^{\alpha} u=0, \quad \text { on } \partial \Omega,
\end{gathered}
$$

where $1<p<q^{*},|\alpha|<4$, and $\varphi \in C^{2}(\bar{\Omega})$. For this problem, we prove the existence of weak solution under the following assumptions:

(A1) $f \in L^{\infty}(\Omega)$,

(A2) $f^{+}:=\max \{f(x), 0\}$ is not identically zero.

Let $K:=H_{0}^{2}(\Omega)$ be equipped with the scalar product $(u, v)_{K}:=\int_{\Omega} \Delta u \Delta v \rho d x$, with $\rho(x):=$ $e^{\varphi(x)}$. Then the induced norm on $K$ is $\|u\|_{K}=\left(\int_{\Omega}|\Delta u|^{2} \rho d x\right)^{1 / 2}$. Notice that $K$ is compactly embedded in $L_{\rho}^{p+1}(\Omega)$, where

$$
\|u\|_{L_{\rho}^{p+1}}=\left(\int_{\Omega}|u|^{p+1} \rho d x\right)^{1 /(p+1)} .
$$

Now we have the following theorem.

Theorem 4.1. Assume that (A1)-(A2) hold and $1<p<q^{*}$, then there exists a nonnegative (nontrivial) solution of (4.1).

Proof. The Euler functional of (4.1) is given by

$$
I[u]:=\frac{1}{2} \int_{\Omega}|\Delta u|^{2} \rho d x-\frac{1}{p+1} \int_{\Omega} f(x)|u|^{p+1} \rho d x,
$$

that is, the conditional critical points of $I$ are weak solutions of (4.1):

$$
\begin{aligned}
\lim \frac{I[u+t v]-I[u]}{t} & =\int_{\Omega} \Delta u \Delta v \rho d x-\int_{\Omega} f(x)|u|^{p-1} u v \rho d x \\
& =\int_{\Omega} \Delta(\rho \Delta u) v d x-\int_{\Omega} f(x)|u|^{p-1} u v \rho d x \\
& =\int_{\Omega}\left(\Delta^{2} u+\left(\Delta \varphi+|\nabla \varphi|^{2}\right) \Delta u+2 \nabla \varphi \cdot \nabla \Delta u-f(x)|u|^{p-1} u\right) v \rho d x
\end{aligned}
$$

Now let

$$
u=r v(x), \quad r \in \mathbb{R} \backslash\{0\}, v \in \Gamma,
$$

where $\Gamma=\left\{v \in K: \int_{\Omega} f(x)|v|^{p+1} \rho d x>0,(v, v)_{K}=1\right\}$, that is, we take the fibering functional $H(v)=\|v\|_{K}^{2}=\int_{\Omega}|\Delta v|^{2} \rho d x$. Let

$$
E(r, v):=I[r v]=\frac{r^{2}}{2} \int_{\Omega}|\Delta v|^{2} \rho d x-\frac{|r|^{p+1}}{p+1} \int_{\Omega} f|v|^{p+1} \rho d x
$$


If we substitute from (4.5) into (4.6), we obtain

$$
E(r, v)=\frac{r^{2}}{2}-\frac{|r|^{p+1}}{p+1} \int_{\Omega} f|v|^{p+1} \rho d x .
$$

Now the bifurcation equation $E_{r}^{\prime}(r, v)=0$ takes the form $r-r|r|^{p-1} \int_{\Omega} f|v|^{p+1} \rho d x=0$. The nontrivial roots of this equation are

$$
r_{1}(v)=-r_{2}(v)=\left(\int_{\Omega} f|v|^{p+1} \rho d x\right)^{-2 /(p-1)} .
$$

Thus we define the functional $\widetilde{E}$ as

$$
\widetilde{E}(v):=E\left(r_{i}(v), v\right)=\left(\frac{1}{2}-\frac{1}{p+1}\right)\left(\int_{\Omega} f|v|^{p+1} \rho d x\right)^{-2 /(p-1)}, \quad i=1,2,
$$

and we let $\widehat{E}(v):=\int_{\Omega} f|v|^{p+1} \rho d x$. It is clear that the conditional critical points of the $\widetilde{E}$ and $\hat{E}$ are the same. In order to find the conditional critical points of $\hat{E}$, we will use the following lemmas.

LEMMA 4.2. Let

$$
M_{0}:=\sup \{\widehat{E}(v) \mid v \in \Gamma\}
$$

then $0<M_{0}<\infty$ and every maximizing sequence of (4.10) is bounded in $K$.

Proof. From (A2) we know that $M_{0}>0$. For the second inequality, let $0<\rho_{0}:=\min \{\rho(x) \mid$ $x \in \bar{\Omega}\}$. Thus, for all $v \in \Gamma$, we have

$$
\begin{aligned}
|\hat{E}(v)| & =\left.\left|\int_{\Omega} f\right| v\right|^{p+1} \rho d x \mid \leq\|f\|_{\infty}\|\rho\|_{\infty}\|v\|_{p+1}^{p+1} \\
& \leq c \rho_{0}^{p+1}\|\Delta v\|_{2}^{p+1} \leq c\left(\int_{\Omega}|\Delta v|^{2} \rho d x\right)^{p+1}=c
\end{aligned}
$$

where $c$ is a constant. This means that $\hat{E}(v)$ is bounded from above on $\Gamma$ and $M<\infty$.

Lemma 4.3. There exists a minimizer $0 \leq \bar{v} \in K$ for (4.10).

Proof. Let $\left\{v_{n}\right\}$ be a maximizing sequence for (4.10). Then, from boundedness of $\left\{v_{n}\right\}$ in $K$ and compactness of the embedding $K \subset L_{\rho}^{p+1}(\Omega)$, we can suppose that there exists $\bar{v} \in K$ such that $\left\{v_{n}\right\}$ converges weakly to $\bar{v}$. Since the norm is lower semicontinuous, we have $H(\bar{v}) \leq \liminf \left\|v_{n}\right\|_{K}=1$. Since $\widehat{E}$ is continuous, $\widehat{E}\left(v_{n}\right) \rightarrow \widehat{E}(\bar{v})=M_{0}$. If $H(\bar{v})=0$, then $\bar{v}=0$ and $\hat{E}(\bar{v})=0$, which is a contradiction. We also show that $H(\bar{v})=1$. If not, then we must have $0<H(\bar{v})<1$. Hence, for suitable $k>1 H(k \bar{v})=1$ and

$$
\widehat{E}(k \bar{v})=k^{p+1} \hat{E}(\bar{v})=k^{p+1} M_{0}>M_{0},
$$

which is a contradiction to (4.10). Finally, we have $\bar{v} \in K$ and

$$
H(|\bar{v}|)=H(\bar{v})=1, \quad \widehat{E}(|\bar{v}|)=\widehat{E}(\bar{v})=M_{0},
$$


3416 Solutions for polyharmonic and biharmonic equations

therefore we can take $\bar{v} \geq 0$. Now, let $u=r(\bar{v}) \bar{v}$, where

$$
r(\bar{v})=\left(\int_{\Omega} f|v|^{p+1} \rho d x\right)^{-2 /(p-1)}>0 .
$$

Then $u$ is a nonnegative nontrivial weak solution of (4.1).

Remark 4.4. Notice that here again Lyusternik-Shnirelman theory can be applied to the functional $\hat{E}(v)$ on the unit sphere in $K_{0}$. According to this theory, $\hat{E}(v)$ has countably many alternating conditional critical points $v_{1}, v_{2}, \ldots, v_{k}, \ldots$ such that $\|v\|_{K_{0}}=1$ and $\tilde{E}\left(v_{k}\right) \rightarrow 0$ as $k \rightarrow \infty$. Therefore, the problem (4.1) has countably many alternating solutions $\left\{u_{k}\right\}: u_{k}(x)= \pm\left(\int_{\Omega} f\left|v_{k}\right|^{p+1} \rho d x\right)^{-2 /(p-1)} v_{k}(x)$.

Remark 4.5. Consider the equation

$$
\Delta^{2} u+\left(\Delta \varphi+|\nabla \varphi|^{2}\right) \Delta u+2 \nabla \varphi \cdot \nabla \Delta u=\varepsilon f(x)|u|^{p-1} u+\delta g(x)|u|^{q-1} u+h(x),
$$

with

$$
D^{\alpha} u=0, \quad \text { on } \partial \Omega \text {, }
$$

where $|\alpha|<4,1<p<q<q^{*}, h \in K^{*}, 0 \leq f, g \in L^{\infty}(\Omega), 0<b_{0} \leq g(x)$. For the problem (4.15)-(4.16), we can apply the techniques presented in the proof of Theorem 3.4 to the functional

$$
\begin{aligned}
I[u]:= & \frac{1}{2} \int_{\Omega}|\Delta u|^{2} \rho d x-\frac{\varepsilon}{p+1} \int_{\Omega} f(x)|u|^{p+1} \rho d x \\
& -\frac{\delta}{q+1} \int_{\Omega} g(x)|u|^{q+1} \rho d x-\int_{\Omega} h u \rho d x
\end{aligned}
$$

and arrive to the following theorem.

THEOREM 4.6. Under the above condition, the following results hold.

(1) If $\varepsilon=\delta=-1$, then for every $h \in K^{*},(4.15)$-(4.16) has one weak solution.

(2) If $\varepsilon=\delta=1$, then for sufficiently small $\|h\|_{*}$, (4.15)-(4.16) has three weak solutions.

(3) If $\varepsilon=-\delta=1$, then for sufficiently small $\|h\|_{*}$, (4.15)-(4.16) has three weak solutions.

\section{Acknowledgment}

Both authors would like to thank Sharif University of Technology for supporting this research.

\section{References}

[1] S. Agmon, A. Douglis, and L. Nirenberg, Estimates near the boundary for solutions of elliptic partial differential equations satisfying general boundary conditions. I, Comm. Pure Appl. Math. 12 (1959), 623-727.

[2] T. Bartsch, M. Schneider, and T. Weth, Multiple solutions of a critical polyharmonic equation, J. reine angew. Math. 571 (2004), 131-143. 
[3] H. Brézis and L. Nirenberg, Positive solutions of nonlinear elliptic equations involving critical Sobolev exponents, Comm. Pure Appl. Math. 36 (1983), no. 4, 437-477.

[4] L. C. Evans, Partial Differential Equations, Graduate Studies in Mathematics, vol. 19, American Mathematical Society, Rhode Island, 1998.

[5] H.-Ch. Grunau, The Dirichlet problem for some semilinear elliptic differential equations of arbitrary order, Analysis 11 (1991), no. 1, 83-90.

[6] H.-Ch. Grunau and G. Sweers, Classical solutions for some higher order semilinear elliptic equations under weak growth conditions, Nonlinear Anal. 28 (1997), no. 5, 799-807.

[7] - Positivity for equations involving polyharmonic operators with Dirichlet boundary conditions, Math. Ann. 307 (1997), no. 4, 589-626.

[8] S. Li and Z. Liu, Perturbations from symmetric elliptic boundary value problems, J. Differential Equations 185 (2002), no. 1, 271-280.

[9] S. Luckhaus, Existence and regularity of weak solutions to the Dirichlet problem for semilinear elliptic systems of higher order, J. reine angew. Math. 306 (1979), 192-207.

[10] H. Mâagli, F. Toumi, and M. Zribi, Existence of positive solutions for some polyharmonic nonlinear boundary-value problems, Electron. J. Differential Equations 2003 (2003), no. 58, 1-19.

[11] S. I. Pohozaev, Eigenfunctions of the equation $\Delta u+\lambda f(u)=0$, Soviet Math. Dokl. 6 (1965), $1408-1411$.

[12] On the global fibration method in nonlinear variational problems, Proc. Steklov Inst. Math. 219 (1997), no. 4, 281-328.

[13] The fibering method in nonlinear variational problems, Topological and Variational Methods for Nonlinear Boundary Value Problems (Cholín, 1995), Pitman Res. Notes Math. Ser., vol. 365, Longman, Harlow, 1997, pp. 35-88.

[14] S. I. Pohozaev and P. Drábek, Positive solutions for the p-Laplacian: application of the fibering method, Proc. Roy. Soc. Edinburgh Sect. A 127 (1997), no. 4, 703-726.

[15] S. I. Pohozaev and L. Véron, Multiple positive solutions of some quasilinear Neumann problems, Appl. Anal. 74 (2000), no. 3-4, 363-391.

[16] P. Pucci and J. Serrin, A general variational identity, Indiana Univ. Math. J. 35 (1986), no. 3, 681-703.

[17] Critical exponents and critical dimensions for polyharmonic operators, J. Math. Pures Appl. (9) 69 (1990), no. 1, 55-83.

[18] V. Rădulescu and D. Smets, Critical singular problems on infinite cones, Nonlinear Anal. 54 (2003), no. 6, 1153-1164.

[19] G. Tarantello, On nonhomogeneous elliptic equations involving critical Sobolev exponent, Ann. Inst. H. Poincaré Anal. Non Linéaire 9 (1992), no. 3, 281-304.

[20] F. Tomi, Über elliptische Differentialgleichungen 4. Ordnung mit einer starken Nichtlinearität, Nachr. Akad. Wiss. Göttingen Math.-Phys. Kl. II (1976), no. 3, 33-42.

M. Hesaaraki: Department of Mathematical Sciences, Sharif University of Technology, P.O. Box 11365-9415, Tehran, Iran

E-mail address: hesaraki@sina.sharif.edu

B. Raessi: Department of Mathematical Sciences, Sharif University of Technology, P.O. Box 113659415, Tehran, Iran

E-mail address: raessi@math.sharif.edu 


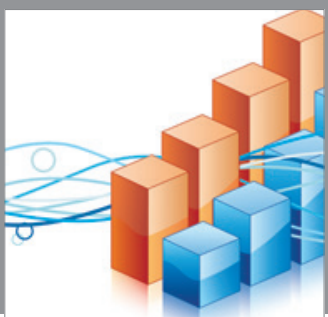

Advances in

Operations Research

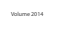

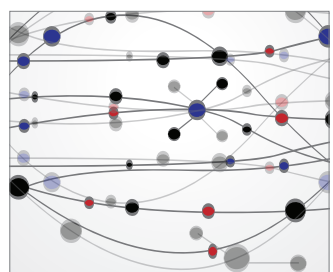

\section{The Scientific} World Journal
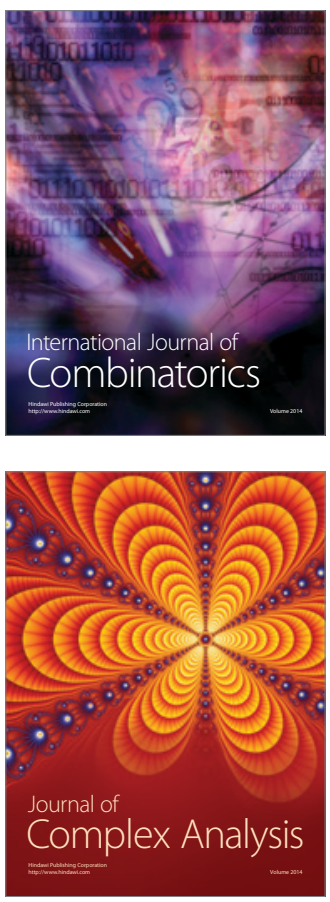

International Journal of

Mathematics and

Mathematical

Sciences
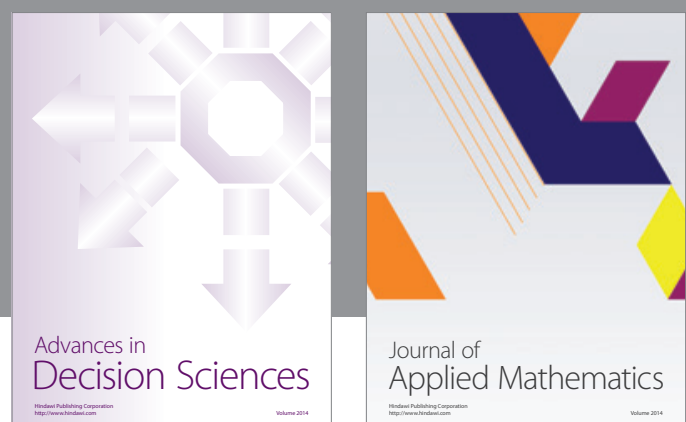

Journal of

Applied Mathematics
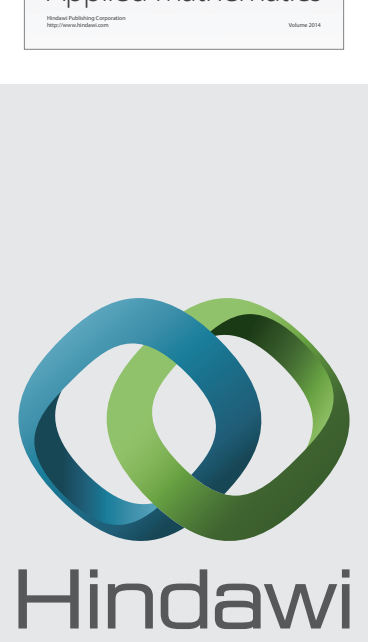

Submit your manuscripts at http://www.hindawi.com
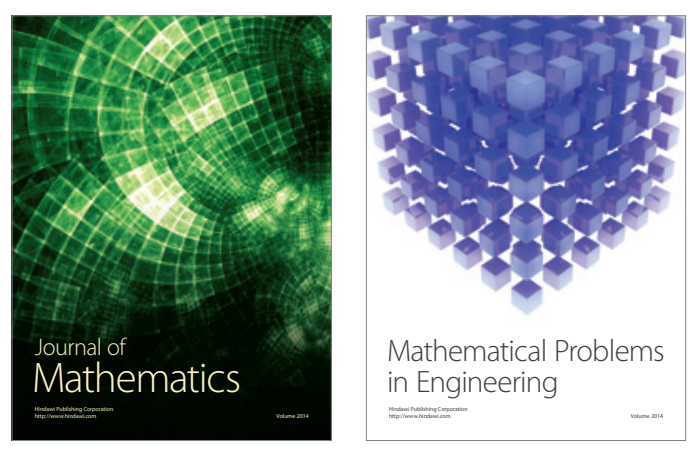

Mathematical Problems in Engineering
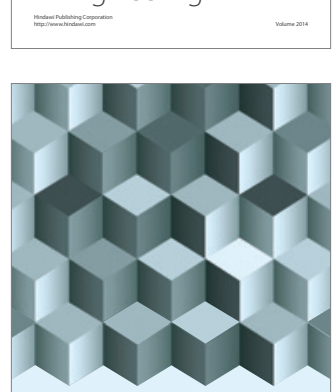

Journal of

Function Spaces
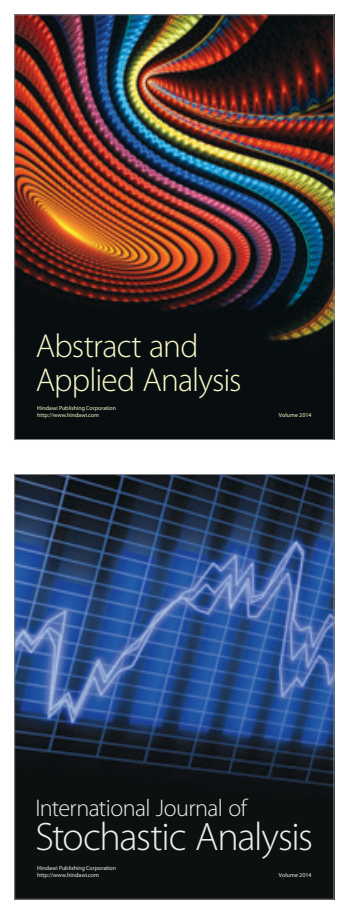

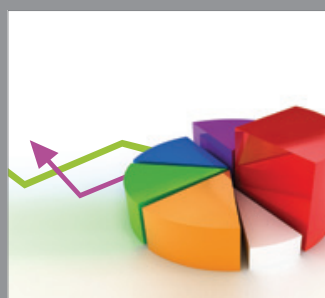

ournal of

Probability and Statistics

Promensencen
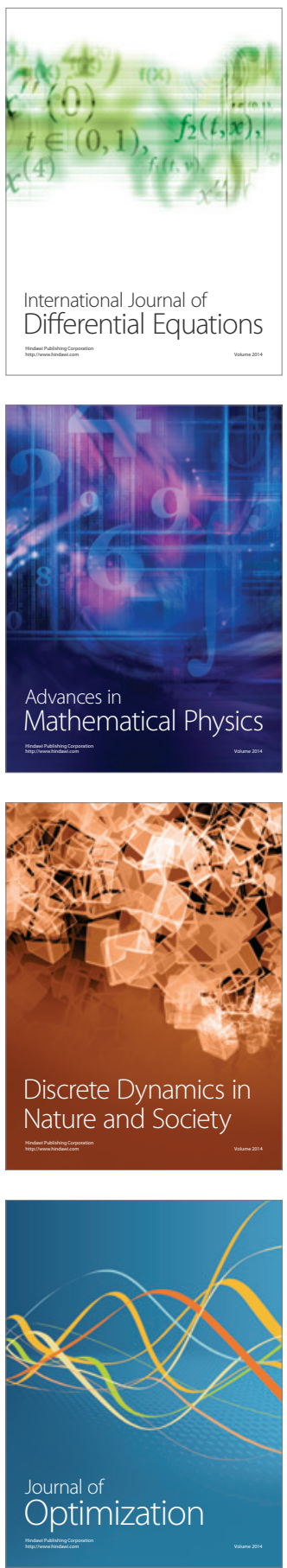